\title{
Combinations of laminin 5 with PTEN, p-EGFR and p-Akt define a group of distinct molecular subsets indicative of poor prognosis in patients with non-small cell lung cancer
}

\author{
SHE-JUAN AN, QIU-XIONG LIN, ZHI-HONG CHEN, JIAN SU, HUA CHENG, ZHI XIE, XU-CHAO ZHANG, \\ HAI-YU ZHOU, YING HUANG, SHI-LIANG CHEN, WEI-BANG GUO and YI-LONG WU
}

Guangdong Lung Cancer Institute, Medical Research Center of Guangdong General Hospital, Guangdong Academy of Medical Sciences, Guangzhou 510080, P.R. China

Received February 17, 2012; Accepted May 10, 2012

DOI: $10.3892 / \mathrm{etm} .2012 .577$

\begin{abstract}
Laminin $5(\operatorname{Ln} 5)$ is an extracellular matrix protein that plays an important role in cell migration and tumor invasion. This study explored the expression of Ln5 and the role of its relationships with PTEN, phospho-EGFR (p-EGFR) and phospho-Akt (p-Akt) in the prognosis of patients with non-small cell lung cancer (NSCLC). The protein expression of Ln5, PTEN, p-EGFR and p-Akt was assessed by immunohistochemical analysis, and their relationships to prognosis were analyzed. Protein expression of Ln5, p-EGFR and p-Akt was detected in 61.2 (60/98), 60.2 (59/98) and 45.3\% (43/95) of patients with NSCLC, respectively. Loss of PTEN expression was found in $67.7 \%$ of tumors (65/96). Ln5 expression was related to patient gender, histology and p-Akt expression $\left(\chi^{2}=3.901,4.549\right.$ and 6.985, respectively; $\mathrm{P}=0.048,0.033$ and 0.008 , respectively). Patients with positive Ln5 expression had marginally poorer survival than Ln5-negative patients (median survival time 56.4 months vs. not reached; $\chi^{2}=3.346 ; \mathrm{P}=0.067$ ). Overall survival was significantly different in patients with positive Ln5 expression combined with loss of PTEN, positive p-EGFR expression or positive p-Akt expression. Cox regression analysis showed that stage, co-expression of Ln5 and p-Akt, and PTEN were the three most independent prognostic factors for patients with NSCLC $\left(\chi^{2}=27.906 ; \mathrm{P}<0.0005\right)$. The results highlight the complex relationships between extracellular matrix proteins and key signaling pathway molecules in tumorigenesis. Changes in the expression of Ln5 plus PTEN,
\end{abstract}

Correspondence to: Professor Yi-Long Wu, Guangdong Lung Cancer Institute, Medical Research Center of Guangdong General Hospital, Guangdong Academy of Medical Sciences, Guangdong, Guangzhou 510080, P.R. China

E-mail: syylwu@live.cn

Abbreviations: Ln5, laminin 5; NSCLC, non-small cell lung cancer; EGFR, epidermal growth factor receptor; ECM, extracellular matrix

Key words: lung cancer, laminin 5, PTEN, phospho-epidermal growth factor receptor, phospho-Akt
p-EGFR or p-Akt define a distinct subset of lung cancers. Patients with such cancers have poorer survival and require early treatment that impacts survival.

\section{Introduction}

Lung cancer is the leading cause of cancer-related death in the United States and throughout the world in both men and women $(1,2)$. Due to diagnosis in late disease stages and the poor treatment efficacy of metastatic disease, overall survival is $>15 \%$ and has not improved substantially in the last 30 years (3). Tyrosine kinase inhibitors (TKIs) targeting epidermal growth factor receptor (EGFR), including gefitinib and erlotinib, have become the standard first-line therapy for patients with advanced non-small cell lung cancers (NSCLCs) harboring activating EGFR mutations $(4,5)$. However, almost all patients eventually develop resistance to EGFR TKIs.

Laminin 5 (Ln5, also known as laminin 332) is an extracellular matrix (ECM) protein that plays an important role in cell migration and tumor invasion $(6,7)$. It has a cruciform structure with one long arm and three short arms. The coiled-coil structure of the long arm is formed by three chains $(\alpha 3, \beta 3$ and $\gamma 2)$ covalently linked via interchain disulfide bonds (8). The rod-like regions in the short arms are composed of EGF-like repeats intercalated with globular domains (9). Given the multi-domain architecture of Ln5, it seems conceivable that other receptors in addition to integrins interact with one of its many potential ligand sites to mediate its diverse cellular functions, including activation of EGFR signaling.

EGFR is a key mediator of oncogenesis in NSCLCs, with its activation inducing tumor proliferation and growth, angiogenesis, invasion and metastasis, and inhibiting apoptosis (10). Activation of Akt (murine thymoma viral oncogene homolog) is one of the mechanisms that mediate the effects of EGFR (11). The Akt pathway regulates diverse biological functions (12). The tumor-suppressor gene PTEN negatively regulates the PI3K/Akt signaling pathway (13).

Interactions between Ln5 and PTEN, phospho-EGFR (p-EGFR) and phospho-Akt (p-Akt) in patients with NSCLC are not well understood at the clinical level. Thus, we measured 
the expression levels of Ln5, PTEN, p-EGFR and p-AKT and analyzed their relationships to prognosis. The results may be helpful for determining the relationships between these factors and potential prognostic factors and may have important implications for individualized therapy.

\section{Materials and methods}

Patient selection. A total of 98 tissue samples were obtained from the tumor bank of Guangdong Lung Cancer Institute (Guangzhou, China) between 2004 and 2006. All specimens were collected after informed consent was obtained. Data on histological type, clinical stage, smoking status, gender and patient age were collected from medical records. Patients were followed through telephone calls or re-examination of records by the hospital follow-up group. Survival was determined from the date of surgical resection until the date of the last time of follow-up (August 1, 2011). The median follow-up time for overall survival was 53.9 months. A total of 46/98 patients in the study $(46.9 \%)$ died during this period.

Antibody selection and immunohistochemistry. We immunohistochemically examined the protein expression of Ln5, PTEN, p-EGFR and p-Akt in 98 frozen tumor samples from the lung cancer bank. Frozen sections (6 to $8 \mu \mathrm{m}$ thick) were prepared, immediately fixed through incubation in cold methanol for $10 \mathrm{~min}$ and then air-dried. These sections were washed in PBS. Subsequently, endogenous peroxidase activity was blocked through incubation with $3 \% \mathrm{H}_{2} \mathrm{O}_{2}$ for $10 \mathrm{~min}$. Sections were incubated overnight at $4^{\circ} \mathrm{C}$ with antibodies against human Ln5 (dilution 1:1,000; Abcam, Cambridge, UK), PTEN (1:80; Fuzhou Maixin Biotechnology Development, Co., Fuzhou, China), phospho-EGFR (Tyr 1086, 1:100; Cell Signaling Technology, Inc., Danvers, MA, USA) and phosphoAkt (Ser 473, 1:100; Cell Signaling Technology, Inc.), then rinsed with $\mathrm{PBS}$ and subsequently treated with components of a ready-to-use Histostain-Plus secondary antibody kit (Shenzhen Jingmei Biotech, Co, Ltd., Shenzhen, China). Finally, the chromogenic substrate 3,3-diaminobenzidine tetrahydrochloride (DAB) was added. The specimens were counterstained with hematoxylin, mounted and examined with a BX50 light microscope (Olympus). Negative controls were treated with PBS instead of primary antibody to verify specificity.

Two authors (Q.X. Lin and S.J. An), who were blinded to clinical details of the specimens, reviewed all of the slides simultaneously. When the opinions of the two evaluators differed, a consensus was reached through discussion.

Assessment of immunohistochemistry. The intensity of tissue staining was scored on a semi-quantitative $0-3$ scale (with 0 representing no staining and 3 the strongest staining). Aberrant expression of PTEN, p-EGFR and p-AKT was judged by cytoplasmic staining. The expression pattern of Ln5 was assessed as follows: 3, continuous linear immunostaining in the basement membrane; 2 , continuous and discontinuous linear immunostaining in the same specimen; 1 , discontinuous linear immunostaining; and 0, no immunostaining. Immunohistochemistry results were then dichotomized as negative ( score $=0$ ) or positive (score $>0$ ).
Table I. Patient characteristics.

\begin{tabular}{lcc}
\hline Variable & Group & $\mathrm{n}(\%)$ \\
\hline Gender & Male & $56(57.1)$ \\
& Female & $42(42.9)$ \\
Age (years) & Mean & 56.7 \\
Cigarette smoking & Range & $26-77$ \\
& No & $60(61.2)$ \\
Histological status & Yes & $38(38.8)$ \\
& AC & $62(63.3)$ \\
& SCC & $24(24.5)$ \\
Stage & ASC & $5(5.1)$ \\
& LCC & $7(7.1)$ \\
& I & $47(48.0)$ \\
Follow-up status & II & $14(14.3)$ \\
Total & III & $27(27.6)$ \\
& IV & $10(10.2)$ \\
& Survival & $52(53.1)$ \\
& Death & $46(46.9)$ \\
& & 98
\end{tabular}

AC, adenocarcinoma; SCC, squamous cell carcinoma; ASC, adenosquamous carcinoma; LCC, large cell carcinoma.

Statistical analysis. The relationships between Ln5 and clinical parameters and PTEN, p-EGFR and p-Akt expression levels were analyzed by the $\chi^{2}$ test. Kaplan-Meier survival curves and the log-rank test were used to analyze overall survival. A Cox regression model was used to analyze the relationships between the influencing factors and patient prognosis.

\section{Results}

Patients and protein expression. All patients enrolled in this study had histologically confirmed NSCLC (Table I). Ln5 and p-EGFR expression levels were successfully measured in 98 patients (Fig. 1). Detection procedures for PTEN and p-Akt failed for two and three slides, respectively. These slides were excluded from further analyses. Ln5, p-EGFR and p-Akt were detected in $61.2(60 / 98), 60.2(59 / 98)$ and $45.3 \%(43 / 95)$ of patients with NSCLC, respectively. Loss of PTEN expression was found in $67.7 \%$ of tumors $(65 / 96)$.

Relationships between Ln5 expression and clinical parameters. We analyzed the relationships between Ln5 expression levels and clinical parameters (Table II). Ln5 expression was related to patient gender and histology. Positive Ln5 expression was more common in males than in females (69.6 vs. $50.0 \%$, respectively; $\chi^{2}=3.901 ; \mathrm{P}=0.048$ ) and less common in adenocarcinomas $(\mathrm{AC})$ than in tumors of other histological types [53.2 vs. $75.0 \%$, respectively; $\chi^{2}=4.549$ (two-sided test); $\mathrm{P}=0.033]$. There was no relationship between Ln5 expression and smoking status or tumor stage (Table II). 
Table II. Relationships between Ln5 and clinical parameters ( $\mathrm{n}=98)$.

\begin{tabular}{|c|c|c|c|c|c|}
\hline Variable & Negative, n (\%) & Positive, n (\%) & $\chi^{2}$ & P-value & OR $(95 \% \mathrm{CI})$ \\
\hline Gender & & & 3.901 & 0.048 & $0.436(0.190-1.001)$ \\
\hline Male & $17(30.4)$ & $39(69.6)$ & & & \\
\hline Female & $21(50.0)$ & $21(50.0)$ & & & \\
\hline Smoking status & & & 0.098 & 0.755 & $1.143(0.495-2.640)$ \\
\hline No & $24(40.0)$ & $36(60.0)$ & & & \\
\hline Yes & $14(36.8)$ & $24(63.2)$ & & & \\
\hline Histology & & & 4.549 & 0.033 & $2.636(1.067-6.513)$ \\
\hline $\mathrm{AC}$ & $29(46.8)$ & $33(53.2)$ & & & \\
\hline Others & $9(25.0)$ & $27(75.0)$ & & & \\
\hline Stage & & & 0.500 & 0.480 & $0.740(0.321-1.705)$ \\
\hline $\mathrm{I}+\mathrm{II}$ & $22(36.1)$ & $39(63.9)$ & & & \\
\hline III+IV & $16(43.2)$ & $21(56.8)$ & & & \\
\hline
\end{tabular}

The $\chi^{2}$ tests were two-sided. AC, adenocarcinoma. OR, odds ratio; CI, confidence interval.

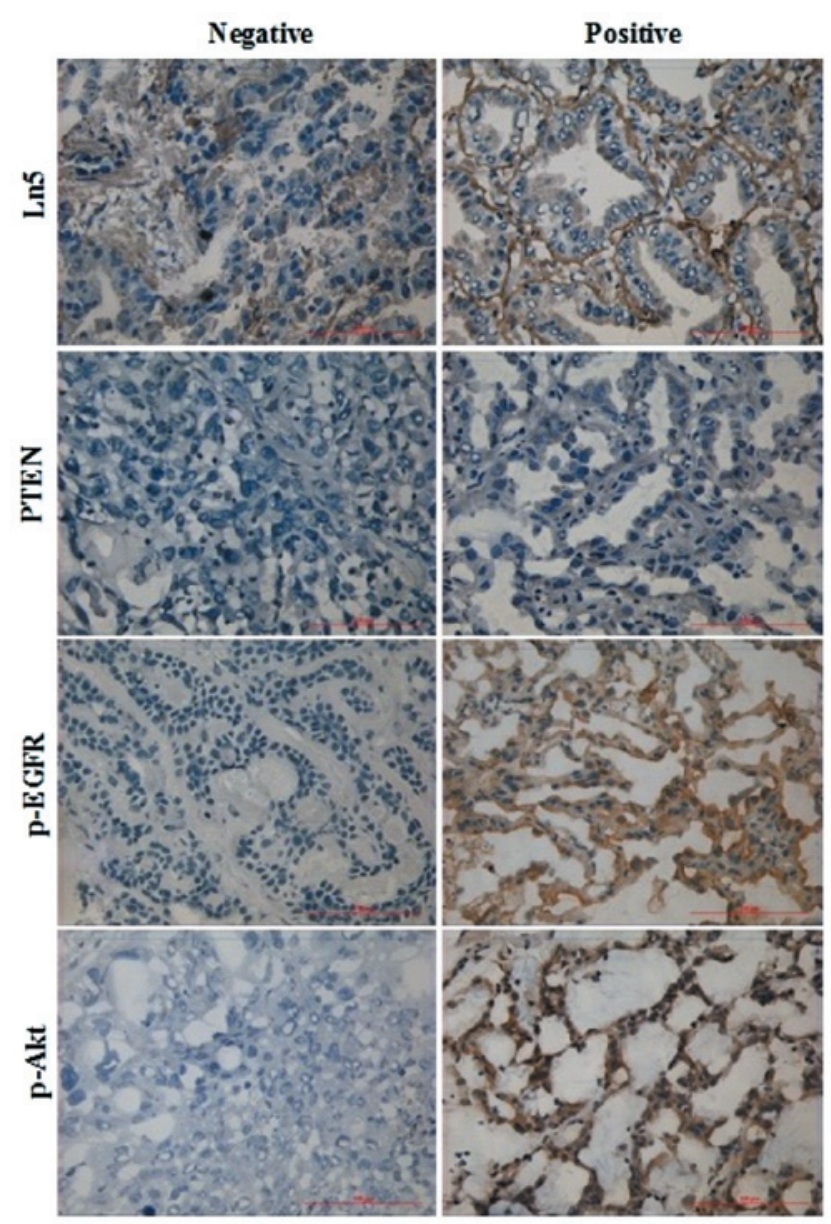

Figure 1. Immunohistochemical analysis of Ln5, PTEN, p-EGFR and p-Akt expression in non-small cell lung cancer tumors (magnification, $\mathrm{x} 400$ ).

Co-alteration of Ln5 and PTEN, p-EGFR and p-Akt. The associations between PTEN, p-EGFR and p-Akt have been well studied. Therefore, we focused on their associations with Ln5 (Table III). Positive Ln5 expression was less common in the p-Akt-positive group than in the p-Akt-negative group [46.5 vs. $73.1 \% ; \chi^{2}=6.985$ (two-sided test); $\left.P=0.008\right]$. No correlations were found between Ln5 and PTEN or p-EGFR.

Prognostic significance of Ln5, PTEN, p-EGFR and p-Akt. Ln5, PTEN, p-EGFR and p-Akt were subjected to survival analysis alone and in combination (Fig. 2). Ln5-positive patients had a marginally shorter survival time than Ln5-negative patients [median survival time 56.4 months vs. not reached; $\chi^{2}=3.346 ; \mathrm{P}=0.067$ (Kaplan-Meier analysis, log-rank test)]. There were no survival differences when PTEN, p-EGFR and p-Akt expression were analyzed alone. However, when combined with Ln5, all of them showed statistically significant differences between positive and negative patients (Fig. 2). Overall survival in NSCLC patients was significantly shorter in patients with positive expression of Ln5 plus loss of PTEN expression, positive expression of Ln5 plus p-EGFR and positive expression of Ln5 plus p-AKT than in other patients. All of the analyzed clinical parameters (gender, smoking status, histology and stage) and molecules (Ln5, PTEN, p-EGFR, p-Akt, Ln5 plus PTEN, Ln5 plus p-EGFR and Ln5 plus p-Akt) were entered into a multivariate analysis. Cox regression analysis showed that stage, Ln5 plus Akt and PTEN were the three most independent prognostic factors in patients with NSCLC $\left(\chi^{2}=27.906 ; \mathrm{P}<0.0005\right.$; forward: Wald; $\mathrm{P}=0.05$, entry; $\mathrm{P}=0.10$, removal) (Table IV).

\section{Discussion}

Interactions between tumor cells and laminin or other components of the extracellular matrix have been shown to play an important role in tumor invasion and metastasis (14). Previous studies have shown that Ln5 is frequently expressed at the invasive front of several types of cancers, including colorectal, gastric, pancreatic and breast adenocarcinomas; uterine, cervical and oral squamous cell carcinomas; malignant melanoma; and small-sized lung adenocarcinomas (maximum dimension, $\leq 2 \mathrm{~cm}$ ) and that overexpression of 
Table III. Relationships between Ln5 and PTEN, p-EGFR and p-Akt.

\begin{tabular}{lccccc}
\hline Variable & Ln5 negative (\%) & Ln5 positive $(\%)$ & $\chi^{2}$ & P-value & OR (95\% CI) \\
\hline PTEN negative & $23(35.4)$ & $42(64.6)$ & 0.847 & 0.357 & $0.665(0.278-1.589)$ \\
positive & $14(45.2)$ & $17(54.8)$ & & & \\
p-EGFR negative & $14(35.9)$ & $25(64.1)$ & 0.226 & 0.634 & $0.817(0.354-1.883)$ \\
positive & $24(40.7)$ & $35(59.3)$ & & & \\
p-Akt negative & $14(26.9)$ & $38(73.1)$ & 6.985 & 0.008 & $0.320(0.136-0.755)$ \\
positive & $23(53.5)$ & $20(46.5)$ & & & \\
\hline
\end{tabular}

The $\chi^{2}$ tests were two-sided. OR, odds ratio; CI, confidence interval.

A

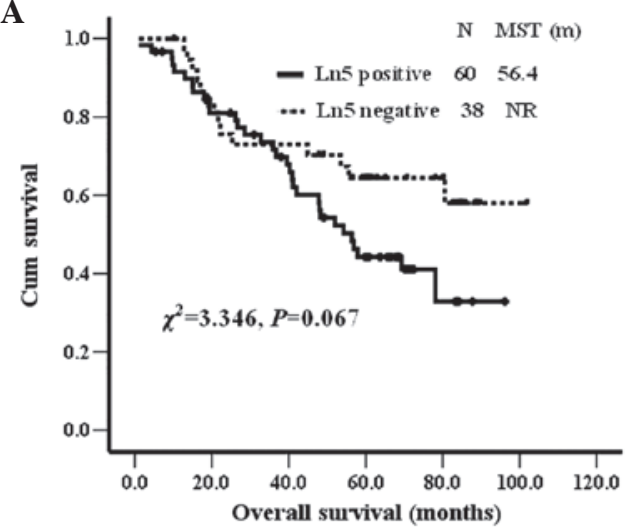

C

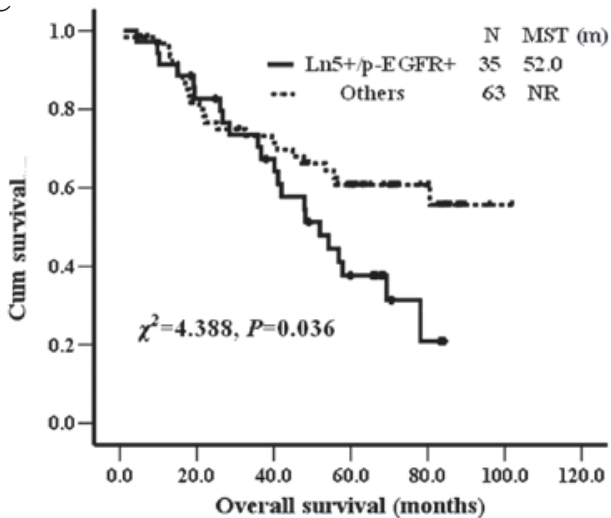

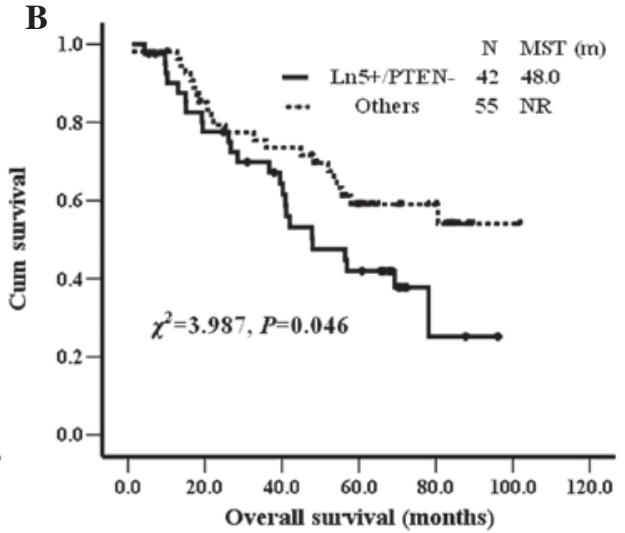

D

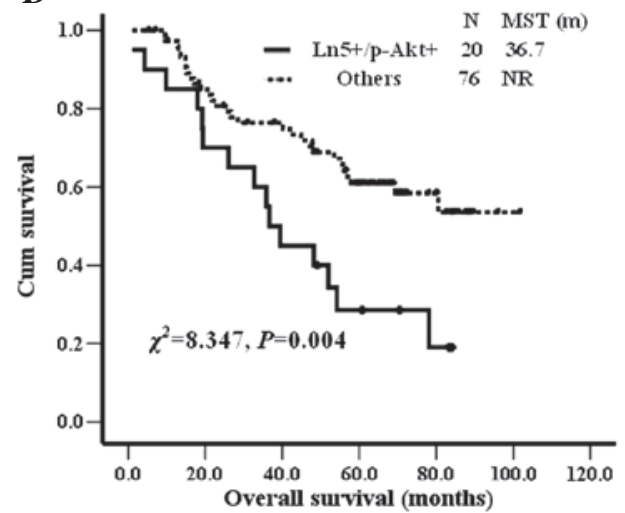

Figure 2. Survival analysis for Ln5 combined with PTEN, p-EGFR and p-Akt. (A) Ln5, (B) Ln5 plus loss of PTEN, (C) Ln5 plus p-EGFR and (C) Ln5 plus p-Akt.

Table IV. Multivariate analysis of overall survival.

\begin{tabular}{lrccc}
\hline Variable(s) & Wald & P-value & OR & $95 \%$ CI \\
\hline Stage & 12.557 & 0.000 & 1.769 & $1.290-2.425$ \\
PTEN & 6.167 & 0.013 & 0.400 & $0.195-0.825$ \\
Ln5 plus p-Akt & 12.840 & 0.000 & 3.384 & $1.737-6.593$ \\
\hline
\end{tabular}

Cox regression analysis, forward: Wald; $\mathrm{P}=0.05$, entry; $\mathrm{P}=0.10$, removal. OR, odds ratio; $\mathrm{CI}$, confidence interval.

Ln5 is associated with poor patient prognosis (15). PTEN, p-EGFR and p-Akt play important roles in tumorigenesis. Yet, the relationships among them in NSCLC patients have not been clarified. Our study revealed for the first time that patients with co-alteration of Ln5 plus PTEN, p-EGFR or p-Akt have poorer overall survival.

It has been reported that gefitinib inhibits the growth of hepatocellular carcinoma cells, Ln5 reduces the ability of gefitinib to inhibit cell growth and the addition of exogenous Ln5 has no effect on p-EGFR but restores p-Akt (16). Another in vitro study performed using A431 cutaneous squamous cell carcinoma cells reported that Ln5- $\gamma 2$ siRNA significantly suppressed EGF-stimulated A431 cell invasion (17). In addition, the introduction of Ln5 may activate a survival signal through EGF-independent EGFR activity in certain types of human lung adenocarcinoma cell lines (18). These results suggest that the signaling pathways mediating carcinoma cell survival, growth and invasion resulting from the action of Ln5 and EGF 
share common downstream signal transduction molecules, such as p-Akt in some tumors. Our study of lung cancer tumor tissues indicates that Ln5 expression has no relationship with p-EGFR, but has a strong relationship with p-Akt.

In the present study, Ln5 expression was negatively correlated with p-Akt expression. p-Akt-negative patients were more frequently positive for Ln5 expression than p-Akt-positive patients. Further analysis of the relationship between p-Akt and p-EGFR demonstrated that p-Akt was positively correlated with p-EGFR. p-Akt is a downstream signal transduction molecule in many pathways. These results suggest that activation of p-Akt primarily results from the upstream activation of p-EGFR and that activation of $p$-Akt can have a negative feedback effect on the expression of Ln5. The biological significance of Ln5 in invading tumor cells is controversial and appears to be somewhat tumor-specific (19). Although Ln5 is negatively correlated with p-Akt, some patients simultaneously express both Ln5 and p-Akt. Individualized treatment is an attractive challenge that may allow for safer and more effective treatment of human disease (20). This phenomenon suggests the existence of complicated relationships between different therapy-targeted genes.

Survival analysis showed that Ln5 expression and loss of PTEN expression were associated with a trend toward worse prognosis in NSCLC patients. p-EGFR and p-Akt had no prognostic significance when analyzed separately. However, when combined with Ln5, they both showed prognostic significance. The multiple Cox regression analysis showed that stage, Ln5 plus p-Akt and PTEN were the three most independent prognostic factors in patients with NSCLC. Stage is a well-known independent prognostic factor. PTEN is one of the key components in the EGFR signaling pathway, and recent studies have suggested that loss of its expression is an independent predictor of poor prognosis in patients with $\operatorname{NSCLC}(13,21)$. Ln5 and p-Akt may play important roles in the development of NSCLC and may represent new predictors of poor prognosis in patients with NSCLC. Our results suggest that Ln5 plays an important role in tumor development, and that altered expression of Ln5 plus PTEN, p-EGFR or p-Akt defines a distinct subset of lung cancers. Patients with these cancers have worse rates of survival and may need to receive earlier treatment that impacts survival.

In conclusion, our study highlights the complex relationships between the extracellular matrix protein Ln5 and key signaling pathway intermediates in tumorigenesis. It also demonstrates the important prognostic significance of Ln5 expression, either alone or in combination with loss of PTEN expression, positive p-EGFR expression or positive p-Akt expression. These changes may serve as unfavorable prognostic factors for NSCLC and may allow the identification of a subset of patients with a poorer prognosis who can be targeted with novel treatments that target Ln5, restore PTEN expression and/or target activated EGFR, Akt or other downstream signal transduction molecules.

\section{Acknowledgements}

We wish to thank Dr Jian-Hua Chen for her excellent follow-up work. This work was supported by grants from the National Natural Science Foundation of China (no. 81101549), the Natural Science Foundation of Guangdong Province (S2011010000792) and the Foundation of Guangdong Science and Technology Department (2006B60101010 and 2007A032000002).

\section{References}

1. Paez JG, Janne PA, Lee JC, et al: EGFR mutations in lung cancer: correlation with clinical response to gefitinib therapy. Science 304: 1497-1500, 2004

2. Bremnesa RM, Sirerab R and Camps C: Circulating tumourderived DNA and RNA markers in blood: a tool for early detection, diagnostics, and follow-up? Lung Cancer 49: 1-12, 2005.

3. Al-Saad S, Donnem T, Al-Shibli K, Persson M, Bremnes RM and Busund LT: Diverse prognostic roles of Akt isoforms, PTEN and $\mathrm{PI} 3 \mathrm{~K}$ in tumor epithelial cells and stromal compartment in non-small cell lung cancer. Anticancer Res 29: 4175-4183, 2009.

4. Mok TS, Wu YL, Thongprasert S, et al: Gefitinib or carboplatinpaclitaxel in pulmonary adenocarcinoma. N Engl J Med 361: 947-957, 2009.

5. Kalikaki A, Koutsopoulos A, Hatzidaki D, et al: Clinical outcome of patients with non-small cell lung cancer receiving front-line chemotherapy according to EGFR and K-RAS mutation status. Lung Cancer 69: 110-115, 2010.

6. Miyazaki K: Laminin-5 (laminin-332): unique biological activity and role in tumor growth and invasion. Cancer Sci 97: 91-98, 2006.

7. Fukai Y, Masuda N, Kato H, et al: Correlation between laminin-5 gamma2 chain and epidermal growth factor receptor expression in esophageal squamous cell carcinomas. Oncology 69: 71-80, 2005.

8. Yurchenco PD and Cheng YS: Self-assembly and calciumbinding sites in laminin A three-arm interaction model. J Biol Chem 268: 17286-17299, 1993.

9. Engel J: Domain organizations of modular extracellular matrix proteins and their evolution. Matrix Biol 15: 295-299, 1996.

10. Lee SY, Kim MJ, Jin G, et al: Somatic mutations in epidermal growth factor receptor signaling pathway genes in non-small cell lung cancers. J Thorac Oncol 5: 1734-1740, 2010.

11. Li F, Liu Y, Chen H, Liao D, Shen Y, Xu F and Wang J: EGFR and COX-2 protein expression in non-small cell lung cancer and the correlation with clinical features. J Exp Clin Cancer Res 30: 27, 2011.

12. Yoshizawa A, Fukuoka J, Shimizu S, et al: Overexpression of phospho-eIF4E is associated with survival through AKT pathway in non-small cell lung cancer. Clin Cancer Res 16: 240-248, 2010.

13. Tang JM, He QY, Guo RX and Chang XJ: Phosphorylated Akt overexpression and loss of PTEN expression in non-small cell lung cancer confers poor prognosis. Lung Cancer 51: 181-191, 2006.

14. Menard S, Castronovo V, Tagliabue E and Sobel ME: New insights into the metastasis-associated $67 \mathrm{kD}$ laminin receptor. J Cell Biochem 67: 155-165, 1997.

15. Moriya Y, Niki T, Yamada T, Matsuno Y, Kondo H and Hirohashi S: Increased expression of laminin-5 and its prognostic significance in lung adenocarcinomas of small size. An immunohistochemical analysis of 102 cases. Cancer 91: 1129-1141, 2001.

16. Giannelli G, Azzariti A, Fransvea E, Porcelli L, Antonaci S and Paradiso A: Laminin-5 offsets the efficacy of gefitinib ('Iressa') in hepatocellular carcinoma cells. Br J Cancer 91: 1964-1969, 2004.

17. Hamasaki H, Koga K, Aoki M, et al: Expression of laminin $5-\gamma 2$ chain in cutaneous squamous cell carcinoma and its role in tumour invasion. Br J Cancer 105: 824-832, 2011.

18. Kodama K, Ishii G, Miyamoto S, et al: Laminin 5 expression protects against anoikis at aerogenous spread and lepidic growth of human lung adenocarcinoma. Int J Cancer 116: 876-884, 2005.

19. Yuen HW, Ziober AF, Gopal P, et al: Suppression of laminin-5 expression leads to increased motility, tumorigenicity, and invasion. Exp Cell Res 309: 198-210, 2005.

20. Uramoto H, Shimokawa H, Hanagiri T, Kuwano M and Ono M: Expression of selected gene for acquired drug resistance to EGFR-TKI in lung adenocarcinoma. Lung Cancer 73: 361-365, 2011.

21. Wang L, Yue W, Zhang L, Zhao X, Wang Y and Xu S: mTOR and PTEN expression in non-small cell lung cancer: analysis by real-time fluorescence quantitative polymerase chain reaction and immunohistochemistry. Surg Today: 42: 419-425, 2012. 